[Article]

\title{
碳气凝胶的结构重整及其电化学行为
}

\author{
徐子颕 ${ }^{*}$ 吉 涛 赵 蕾 王玮衍 杨春艳 甘礼华 \\ (同济大学化学系, 上海 200092)
}

\begin{abstract}
摘要: 将碳气凝胶(CAs)在金属钠熔体中于 $800^{\circ} \mathrm{C}$ 加热 $3 \mathrm{~h}$, 制得结构重整的碳气凝胶 (RCAs), 并研究了它们 的电化学行为. 采用 $X$ 射线粉末衍射 $(X R D)$ 、激光散射拉曼 (Raman)光谱、气体的吸附与脱附(BET法)、透射电子 显微镜(TEM)以及电化学阻抗谱(EIS)等手段, 对 CAs 和RCAs样品分别进行了表征. 结果表明: 无定形态的碳单 质微粒在金属钠作用下发生结构重排; 所得 RCAs 的比表面积比 CAs 的大约增加 $48 \%$; RCAs 中孔径在 2 和 4 $\mathrm{nm}$ 尺寸处, 呈现比较集中的分布, 孔径小于 $10 \mathrm{~nm}$ 以下的孔体积占其总孔容的 $30 \%$, 是 CAs 的 3 倍. CAs 和 RCAs 样品的电化学测试结果表明: RCAs 内部接触电阻约为 CAs 的 $45 \%$, 并保持了明显的电容特性.
\end{abstract}

关键词: 碳气凝胶; 金属钠熔体; 结构重整; 电化学阻抗谱

中图分类号： O646; O642; 0792

\section{Restructured Carbon Aerogels and Their Electrochemical Performances}

\author{
XU Zi-Jie* Jl Tao ZHAO Lei WANG Wei-Yan YANG Chun-Yan GAN Li-Hua
}

(Department of Chemistry, Tongji University, Shanghai 200092, P. R. China)

\begin{abstract}
Restructured carbon aerogels (RCAs) were obtained by annealing carbon aerogels (CAs) in sodium melt at $800{ }^{\circ} \mathrm{C}$ for $3 \mathrm{~h}$ and the differences in electrochemical performance between the resultant CAs and RCAs were studied. X-ray diffraction (XRD), Raman scattering spectra, gas physisorption (BET), transmission electron microscopy (TEM), and electrochemical impedance spectroscopy (EIS) were used to probe the structures of CAs and RCAs. The studies indicated that the amorphous carbon particles were rearranged in sodium melt, in which the surface area of the resultant RCAs was $48 \%$ larger than that of CAs. Two concentrated pore distributions were observed at the apertures of 2 and $4 \mathrm{~nm}$ in RCAs. The pore volume at apertures below $10 \mathrm{~nm}$ is $30 \%$ of the total volume in RCAs, which is 3 times as many as that in CAs. Electrochemical performances of both CAs and RCAs were investigated and the results indicate that the inner resistance of RCAs is only $45 \%$ of that in the sample of CAs. The results also indicate that RCAs display good performance in capacitance characteristics.
\end{abstract}

Key Words: Carbon aerogel; Sodium melt; Restructure; Electrochemical impedance spectroscopy

\section{1 引 言}

碳气凝胶 $(\mathrm{CAs})$ 是一种由无定形碳纳米颗粒通 过化学键连接构成的三维网络骨架的多孔材料, 它 具有比表面积大、导电性优良等特性, 在新能源电 池和超级电容器方面展现出重要的应用前景. ${ }^{1-6}$

随着人们对 CAs 材料及其应用研究工作的深
入开展, 对该材料性能提出了更高的要求, 希望获 得导电性更好的 CAs 材料. ${ }^{7.8}$ 然而, CAs 的导电性与 该材料的制备方法有很大的相关性. 一般的制备方 法是以有机物作前驱体, 经过溶胶-凝胶过程, 形成 三维的网络骨架结构, 然后通过超临界流体干燥工 艺或经改进的常压干燥工艺对其干燥, 再经过碳化

Received: September 26, 2011; Revised: November 26, 2011; Published on Web: December 6, 2011.

"Corresponding author. Email: xuzijie-tj@126.com; Tel: +86-21-65982654-8430.

The project was supported by the National Natural Science Foundation of China (20973127).

国家自然科学基金(20973127)资助项目

(C) Editorial office of Acta Physico-Chimica Sinica 
处理, 最终获得产品. ${ }^{9-12}$ 由于通过前驱体裂解产生 的纳米碳颗粒由微小的乱碳层的残片, 即碳单质微 粒杂乱无章地堆砌而成, 使得 CAs 的导电性以及电 容性能都不能满足实际应用的要求, 促使人们进一 步开展对 CAs 进行结构优化的研究工作.

对 CAs 石墨化处理是目前提高该材料导电性 的主要途径. 由于无定形碳需要在 $2200{ }^{\circ} \mathrm{C}$ 以上的 高温条件下才能对其直接进行石墨化, 而高温对材 料结构的破坏性极大. 因此, 目前普遍采用催化石 墨化的方法, 在 $1200{ }^{\circ} \mathrm{C}$ 左右的温度条件下, 对 CAs 进行石墨化处理. 在该方法中, 一般将铁、钴、镍、锰 等金属盐与碳的先驱物在溶胶-凝胶过程中共凝胶, 然后, 经过凝胶的干燥过程和碳化处理并在 1000-1200 ${ }^{\circ} \mathrm{C}$ 条件下, 对含有催化剂的 CAs 进行热 处理, 最终得到石墨化的 CAs. ${ }^{13-17}$ 但是, 在金属盐与 碳前驱体的共凝胶化过程中, 由于金属离子的电荷 效应, 改变了原有胶体粒子的表面性质, 使得共凝 胶化的条件变得更加苛刻, 改变了 CAs 原有的包括 孔体积、孔径、孔隙率及比表面积等在内的结构特 征; 另外, 该方法需要使用大量的强酸溶液来去除 残留的金属化合物, 工业化生产时将产生大量的工 业废酸, 对环境不友好. 本文作者先期对无定形碳 在金属钠熔体中的相变进行研究, 结果表明杂乱无 章的碳颗粒在金属钠熔体中发生重排, 并获得一种 在较低温度条件下对无定形碳实施石墨化处理的 方法 ${ }^{18}$ 为本文的研究工作奠定了基础.

本文在金属钠熔体中, 实现了 CAs 骨架上的碳 单质微粒的重排并获得了结构重整的碳气凝胶 (RCAs). 对所得样品分别进行 X 射线粉末衍射 (XRD)、拉曼激光散射(Raman)、气体吸附-脱附(BET 法)、透射电子显微镜(TEM) 以及电化学阻抗谱(EIS) 等方法的表征, 研究了 CAs 在结构重整前后, 其结 构特征和电化学行为的变化. 探讨了由金属钠熔体 引起 CAs 结构改变的机理. 为改善 CAs 材料电化学 特性探索了一条新的途径.

\section{2 实 验}

\section{1 试 剂}

氢氧化钾 $(A R,>96 \%$, 上海强顺化学试剂有限 公司), 间苯二酚( $\mathrm{AR}, \geq 99.5 \%$, 上海新天峰化工有限 公司), 甲醛(AR, $37.0 \%-40.0 \%$, 上海海曲化工有限 公司)、十六烷基三甲基溴化铵(AR, $\geq 99.0 \%$, 中国医 药集团化学试剂公司) 以及钠 $(\mathrm{CP}, \geq 98 \%$, 中国医药
集团化学试剂公司).

\subsection{CAs 的制备}

采用文献 ${ }^{12}$ 报道的制备方法, 分别将间苯二酚 $(5.0 \mathrm{~g})$ 和十六烷基三甲基溴化铵 $(0.083 \mathrm{~g})$ 加入到预 先配制的甲醛 $(6.3 \mathrm{~g})$ 和水 $(7.3 \mathrm{~mL})$ 溶液中, 充分搅拌 至均匀后, 将该溶液移至带玻璃塞的磨口锥形瓶 中, 在 $85^{\circ} \mathrm{C}$ 的水浴中保温 $5 \mathrm{~d}$, 得到半透明的凝胶, 取出后在室温下自然干燥 $2 \mathrm{~d}$, 然后在红外灯下照射 $5 \mathrm{~h}$, 并在 $110^{\circ} \mathrm{C}$ 干燥 $5 \mathrm{~h}$, 再在氮气保护下于 $800^{\circ} \mathrm{C}$ 恒温 $3 \mathrm{~h}$, 最后得到 CAs.

\subsection{CAs 的结构重整}

分别称取 CAs $(5 \mathrm{~g})$ 和金属钠 (4 g), 将它们放置 在带盖的氧化铝坩埚中, 然后将其置于管式炉中, 在氮气气氛中, 以 $5^{\circ} \mathrm{C} \cdot \mathrm{min}^{-1}$ 的升温速率加热至 $800{ }^{\circ} \mathrm{C}$ 并在该温度下保温 $3 \mathrm{~h}$, 然后冷却至室温, 取 出样品并用蒸馏水洗涤至洗液的 $\mathrm{pH}$ 值为 7.0 , 再在 烘箱内于 $70^{\circ} \mathrm{C}$ 干燥, 最终得到 RCAs 样品.

\section{4 表 征}

\subsection{1 材料特性}

使用 Adv D8 型 X射线衍射仪(德国, Bruker)对 样品进行 XRD 表征, 测试条件为 $40 \mathrm{kV}, 40 \mathrm{~mA}, \mathrm{Cu}$ $K_{\alpha}$ 射线; 使用 Invia 型显微共聚焦拉曼光谱仪 (英 国, Rainshaw)对所有样品进行拉曼光谱分析; 使用 TriStar 3000 型比表面积和孔隙度分析仪 (美国, Micromeritics), 采用 Brunauer-Emmett-Teller (BET) 法分析样品的比表面积、孔径分布以及孔结构特征; 使用场发射透射电子显微镜(美国, FEI TECNAI G ${ }^{2}$ S-TWIN F20 型), 对所得样品进行 TEM 形貌表征.

\section{4 .2 电化学行为}

在室温下分别将 CAs、RCAs 和聚四氟乙烯粘 结剂按质量比为 9:1 混合均匀, 压制成直径为 14 $\mathrm{mm}$ 的薄片, 每片的含碳量约为 $15 \mathrm{mg}$, 以镍网为载 体, 将碳片压制于镍网一端, 然后将其在质量浓度 为 $30 \%$ 的氢氧化钾溶液中浸泡 $24 \mathrm{~h}$, 制得测试样品.

以测试样品作为工作电极, 饱和甘录电极作为 参比电极, 镍网作为对电极, 一起组成三电极体系, 电解液为 $30 \%$ (质量分数) 的氢氧化钾水溶液, 采用 CHI600D 电化学工作站对 CAs 和 RCAs 分别采用循 环伏安法和交流阻抗法测试. 循环伏安测试条件: 电势窗口为 -1.0 至 $0 \mathrm{~V}$, 扫描速率为 $5-30 \mathrm{mV} \cdot \mathrm{s}^{-1}$; 交流阻抗测试条件: 测试频率为 $0.1-10^{5} \mathrm{~Hz}$, 起始电 压为 $0 \mathrm{~V}$, 振幅为 $0.005 \mathrm{~V}$. 


\section{3 结果与讨论}

\subsection{CAs 在金属钠熔体中的相变}

图 1 为 CAs 和 RCAs 的 XRD 图谱. 图 $1 \mathrm{a}$ 呈现出 无定形碳的特征衍射谱, 表明经过有机物前驱体的 裂解和碳化过程, CAs 的骨架结构由无定形碳组成. 图 $1 \mathrm{~b}$ 为 RCAs 样品的衍射图谱, 在 $2 \theta$ 衍射角为 $26.085^{\circ}$ 处呈现一个明显的衍射峰, 是(002)晶面的特 征衍射峰. 根据 Bragg 公式, $d=\lambda /(2 \sin \theta)(\lambda$ 为入射 $\mathrm{X}$ 射线波长, $\theta$ 为衍射角), 分别计算得到 $d_{(002)}$ 值为 $0.3414 \mathrm{~nm}, d_{(100)}$ 值为 $0.2098 \mathrm{~nm}$, 不是理想的石墨晶 体, 因为理想石墨单晶的 $d_{(002)}$ 值为 $0.3354 \mathrm{~nm}$. 这种 衍射特征谱表明, 经过在金属钠中的加热处理, CAs 中的无定形碳颗粒(即碳单质微粒)在金属钠中发生 了定向重排, 形成的碳层间距增大, 而增宽的碳层 间距使得电解液离子在其中的渗透与扩散变得更 加容易, 对提高该材料的电容特性十分有利.

显微激光拉曼光谱能够反映分析对象表面及 其以下约 $50 \mathrm{~nm}$ 范围内的信息, 具备分析碳结构的 能力. 图 2 为 CAs 和 RCAs 样品的 Raman 图谱. 图谱 中 $1360 \mathrm{~cm}^{-1}$ 处的峰被称为 $D$ 峰, 表示相同碳原子对 激光的响应模式; $1580 \mathrm{~cm}^{-1}$ 处的峰被称为 $G$ 峰, 表 示不同碳原子对激光的响应模式; $2660 \mathrm{~cm}^{-1}$ 处的峰 被称为 $G^{\prime}$ 峰, 表示相同碳原子和不同碳原子对激光 的综合响应模式, 即反映了处于三维结构中的碳原 子对激光的响应模式. 在图 2 中, CAs 和 RCAs 样品 的 $D$ 峰和 $G$ 峰的积分强度比值 $R\left(I_{\mathrm{D}} / I_{\mathrm{G}}\right)$ 分别为 0.612 和 0.929 , 反映出 RCAs 具有较为完整的晶型结构. 因为其倒数 $\left(R^{-1}\right)$ 与网平面上微晶的平均尺寸或无缺 陷区域成正比关系. ${ }^{19-21}$ 另外, CAs 的 Raman 谱图(如 谱线 $2 \mathrm{a}$ 所示) 中的 $D$ 峰和 $G$ 峰的半峰宽较大, 而且 这两个谱峰重叠部分较多, 没有明显的分离; 在

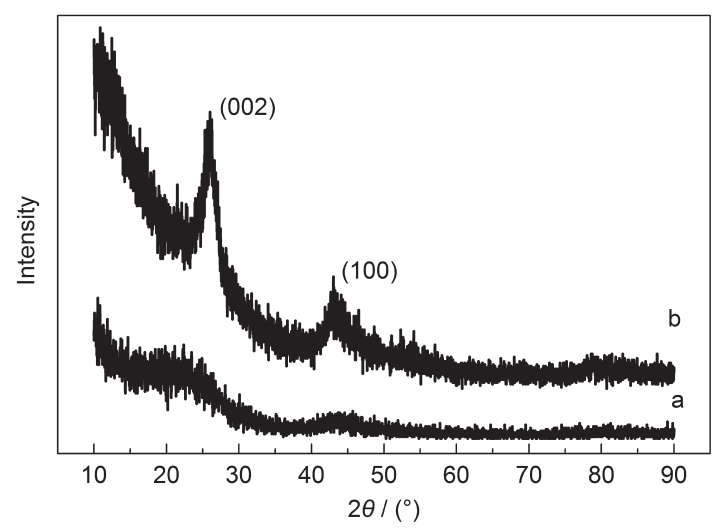

图 1 CAs (a)和 RCAs (b) 的 XRD 图谱 Fig.1 XRD patterns of CAs (a) and RCAs (b)
RCAs 的 Raman 图谱(如谱线 $2 \mathrm{~b}$ 所示)中, $D$ 峰和 $G$ 峰 的半峰宽变窄, $D$ 峰的峰高值减小而 $G$ 峰的峰高值 增大, 并出现了较强的 $G^{\prime}$ 峰. 通过比较 CAs 和 RCAs 的谱图发现它们的谱图有明显的差异, 进一步表明 CAs 经过在金属钠熔体中加热处理后, 其骨架中处 于无定形态单质碳的有序度提高, 从而证实了碳的 相态结构发生变化.

对 CAs 和 RCAs 样品的 XRD、Raman 谱图的分 析结果一致表明, CAs 经过在金属钠熔体中处理后, 所得 RCAs 样品中的碳单质微粒排布呈现出各向异 性的三维晶格特征, 不同于 CAs 中的碳单质微粒杂 乱无章的分布. 当 CAs 与金属钠熔体一起加热时, CAs 中三维网络骨架中存在的连通孔道有利于金属 钠熔体向碳单质微粒表面的渗透与扩散, 改善了无 定形碳发生相变的动力学环境; 另外, 金属钠作为 热的载体, 一方面通过热运动使其本身和碳单质微 粒能够更好地相互接触, 隔绝碳单质与气相的接 触, 减小了它们与气相之间的温度梯度; 另一方面, 碳单质微粒发生重排时释放出的结晶热能够及时 向周围环境释放, 形成有利于碳单质微粒重排的热 力学环境. 当碳单质在金属钠熔体中的最初热力学 活度不同于骨架中碳单质的活度时, 发生了碳单质 的质量迁移和再结晶, 从而导致无定形态的碳单质 微粒发生相变.

\subsection{CAs 在金属钠中的孔结构变化}

CAs 在金属钠熔体中发生相变后, 引起的孔结 构变化反映在所得样品的氮气吸附-脱附低温曲线 上, 如图 3 所示. 图中可见, CAs 和 RCAs 样品的氮气 吸附-脱附低温曲线形状相似, 它们都属于 IV 型孔 结构, 表明 CAs 经过结构重整后, 气凝胶基本的介 孔结构特征并没有明显的改变, 也表明金属钠熔体

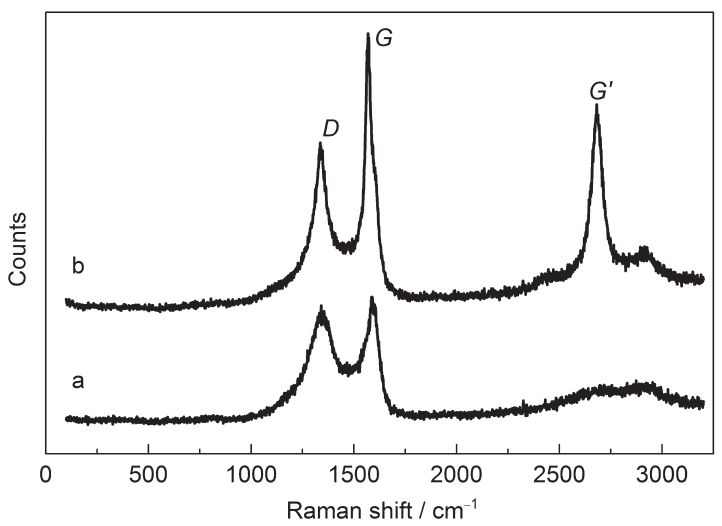

图 2 CAs (a)和 RCAs (b) 的拉曼谱图 Fig.2 Raman spectra of CAs (a) and RCAs (b) 


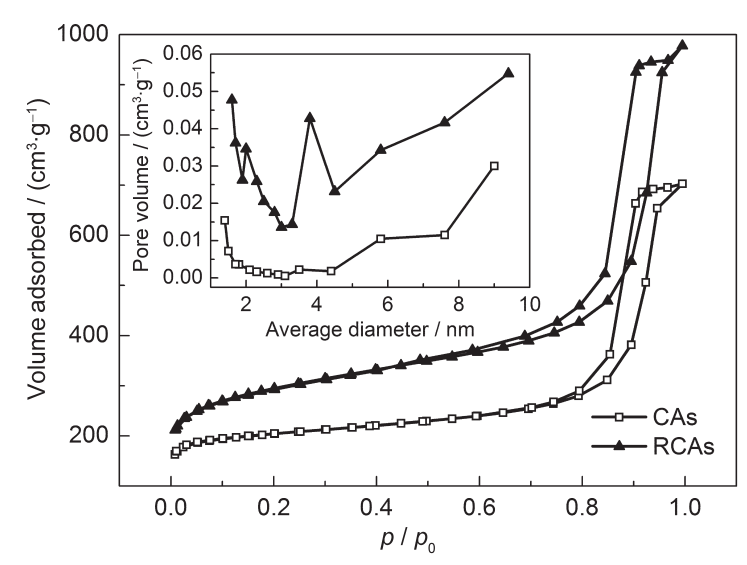

图 3 CAs 和 RCAs 的氮气吸附-脱附等温线和孔径分布 (内插图)

Fig.3 Nitrogen adsorption-desorption isotherms and pore size distributions (inset) of CAs and RCAs

在三维网络骨架中的渗透、扩散以及凝固并没有破 坏气凝胶的基本骨架结构. 但是, 在 RCAs 样品的吸 附-脱附低温曲线中, 可以观察到脱附滞后环在较低 的相对压力处合并, 显示出形成插层孔的吸附-脱附 特征, 而 CAs 样品的吸附-脱附低温曲线中则体现出 堆积微孔的吸附-脱附特征. 插层孔的形成原因是 CAs 骨架中的碳单质微粒在金属钠熔体作用下定向 重排, 使得原本由碳单质微粒堆积而成的封闭微孔 被打开, 形成开放的插层孔.

图 3 中的内插图代表 CAs 和 RCAs 样品的孔径 分布曲线. 从中可见, RCAs 在 2 和 $4 \mathrm{~nm}$ 的孔径处, 分别有较为集中的孔径分布, 而在 CAs 样品中, 没 有观察到在 $10 \mathrm{~nm}$ 以下出现集中的孔径分布. 对它 们的吸附-脱附特性进一步分析可知, RCAs 样品在 $2 \mathrm{~nm}$ 以下以及在 2-4 $\mathrm{nm}$ 之间的单位质量的孔体积 分别为 0.11 和 $0.17 \mathrm{~cm}^{3}$, 而 CAs 在这两个孔径区段 的孔体积分别是 0.030 和 $0.0089 \mathrm{~cm}^{3}$. 另外, 在 RCAs 样品中, 孔径小于 $10 \mathrm{~nm}$ 以下的孔体积占其总孔体

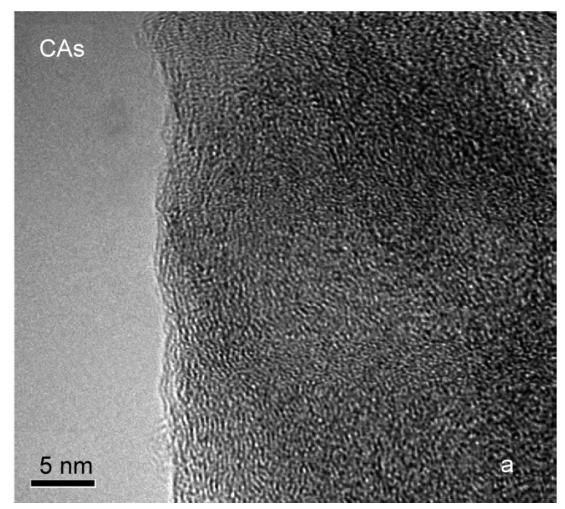

积的比例为 $30 \%$, 而在 CAs 样品中, 这个比例数值 仅为 $10 \%$, 表明 CAs 经过结构重整, 其骨架上原本 由碳单质微粒堆积包围而成的封闭的微孔, 随着碳 单质微粒在金属钠熔体作用下发生重排, 逐渐形成 开放的孔, 使得孔体积显著增多.

$\mathrm{CAs}$ 在金属钠熔体中发生结构重整, 不仅改变 了气凝胶骨架上的孔结构, 而且大大提高了它们的 比表面积. 实验测量发现, RCAs 样品的比表面积为 $1017 \mathrm{~m}^{2} \cdot \mathrm{g}^{-1}$, 而 CAs 样品的比表面积仅为 $688 \mathrm{~m}^{2}$. $\mathrm{g}^{-1}$, 增幅高达 $48 \%$. 因为 CAs 在金属钠熔体中发生结 构重整以后, 碳单质微粒更多的端面被显露出来, 有 益于比表面积的提高, 而介孔体积的增多以及比表 面积的增大对改善材料的电容特性十分有利.

图 4 是 CAs 和 RCAs 样品的 TEM 照片. 由图知, CAs 中碳单质微粒呈现杂乱无章的堆积状态, 其中 分布着由碳微粒所包围的封闭微孔; 在 RCAs 样品 中, 能够清楚地看到碳单质微粒发生的重排以及其 中分布有大量层状孔的形貌. CAs 骨架中的碳单质 微粒在金属钠作用下发生有一定趋向性的重排, 碳 单质微粒之间的间隙有所增大并在其间形成大量 的层状孔.

\subsection{CAs 和 RCAs 的电化学性能}

\subsubsection{CAs 和 RCAs 的循环伏安曲线}

循环伏安测试常用于检测电极/溶液组成的电 化学体系的电容特性. 由 CAs 和 RCAs 分别与电解 质溶液组成电化学体系的循环伏安曲线, 如图 5 所 示. 当扫描速率从 5 到 $30 \mathrm{mV} \cdot \mathrm{s}^{-1}$ 变化时, $\mathrm{CAs}$ 样品 的循环伏安曲线基本没有呈现出类似矩形的电势 窗口, 而且它们的窗口大小几乎没有随着扫描速率 的变化发生改变, 表明此时的 CAs 没有展现出明显 的电容特性变化, 但是, 随着扫描速率的增大, 电势 窗口发生偏转, 表明电子在界面传递过程中所受阻

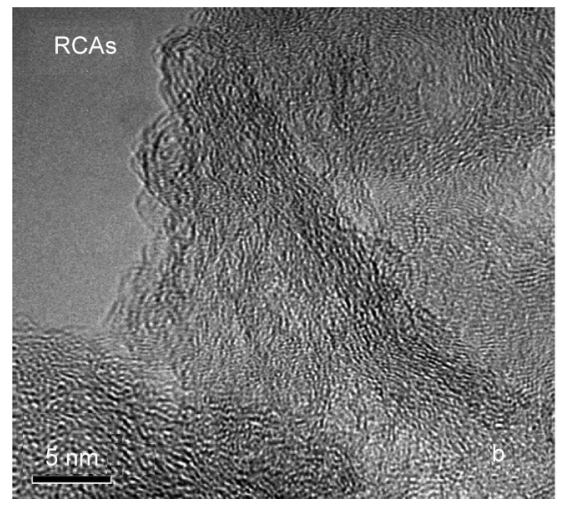

图 4 CAs (a) 和 RCAs (b) 的 TEM 照片

Fig.4 TEM images of CAs (a) and RCAs (b) 

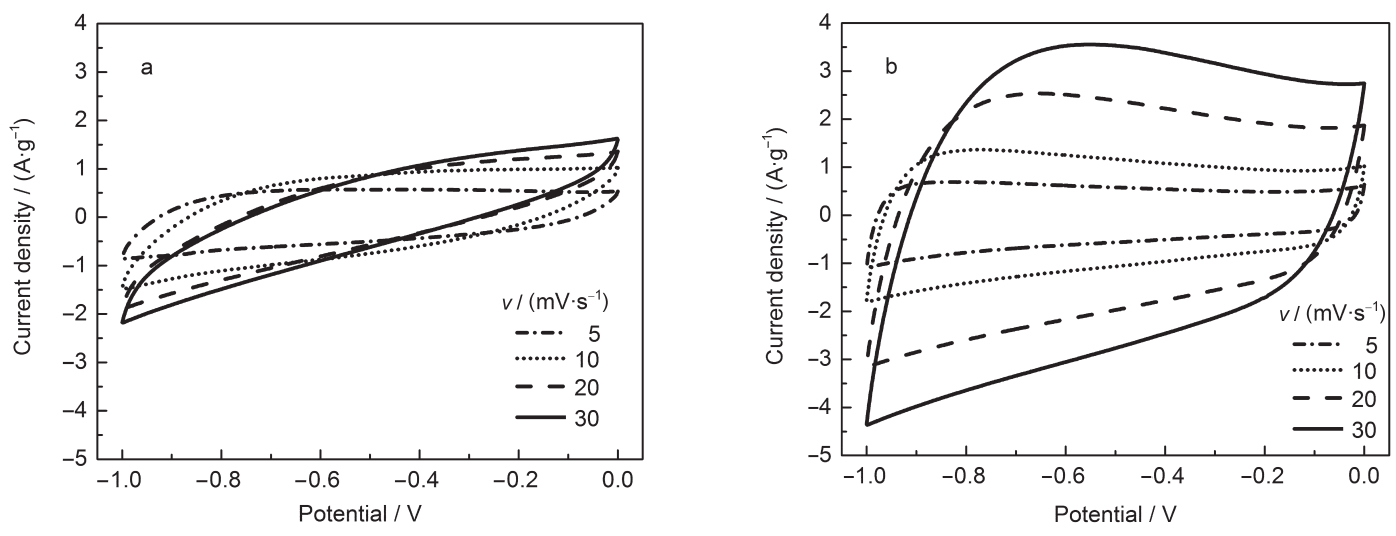

图 5 不同扫描速率(v)下 CAs (a)和 RCAs (b) 的循环伏安曲线

Fig.5 Cyclic voltammetry (CV) curves of CAs (a) and RCAs (b) with different sweep rates (v)

力增大; RCAs 样品同样随着扫描速率的增大, 曲线 中的电势窗口面积逐渐变大, 而且电势窗口的形状 逐渐向矩形发展, 表明此时 RCAs 作为电极, 随着扫 描速率的增大, 电子在界面传递过程中所受阻力没 有明显增大, 而且该电化学体系呈现出较为明显的 电容性能.

根据公式: $C_{m}=\left(I_{\mathrm{a}}+\left|I_{\mathrm{c}}\right|\right) /(2 v m)$, 其中 $I_{\mathrm{a}} 、 I_{\mathrm{c}}$ 分别为电 势窗口对应的阳、阴极电流, $v$ 代表扫描速率, $m$ 为压 制在镍网上电极片的质量, 可以计算得到电极/溶液 电化学体系的比电容 $\left(C_{m}\right)$ 值, 如表 1 所示. 从表中可 见, 在较低扫描速率时, RCAs/溶液的电化学体系的 电容略高于 CAs/溶液的电化学体系的电容值. 但 是, 随着扫描速率的增大, 电荷在 $\mathrm{CAs}$ / 溶液界面传 递过程中所受阻力明显增大, 电容值呈现明显下 降, 而电荷在经过结构重整以后的 RCAs/溶液界面 的电荷传递阻力相对较小, 电容值下降不明显, 表 明该电化学体系的电容行为呈现出较好的稳定性.

\subsubsection{CAs 和 RCAs 的 Nyquist 曲线}

任何一个电化学体系, 在理论上都可以用包含 电阻和电容的等效电路表示该体系的总阻抗, 电阻 和电容分别代表了总阻抗的实部和虚部. 电化学阻 抗谱法可用以分析电极/溶液界面的交流响应特性, 用 Nyquist 曲线表示, 线形的变化可以反映出该电化 学体系动力学过程的变化, 从而间接地反映出电极

表 1 比电容 $\left(C_{m}\right)$ 值随扫描速率的变化

Table 1 Specific capacitance $\left(C_{m}\right)$ value changed with sweep rate

\begin{tabular}{ccc}
\hline \multirow{2}{*}{$v /\left(\mathrm{mV} \cdot \mathrm{s}^{-1}\right)$} & \multicolumn{2}{c}{$C_{m} /\left(\mathrm{F} \cdot \mathrm{g}^{-1}\right)$} \\
\cline { 2 - 3 } & $\mathrm{CAs}$ & $\mathrm{RCAs}$ \\
\hline 5 & 106.9 & 114.5 \\
10 & 80.89 & 111.7 \\
20 & 35.03 & 109.0 \\
30 & 24.37 & 105.0 \\
\hline
\end{tabular}

材料的结构变化对其电容性能的影响.

采用电化学阻抗谱法, 可以得到由 CAs 和 RCAs 分别与电解液组成电化学体系的 Nyquist 曲 线, 如图6所示. 曲线右侧是低频区, 左侧是高频区. 在低频区, Nyquist 曲线中的直线部分反映了电化学 体系阻抗的实部与阻抗的虚部呈现线性相关, 体现 出扩散控制电极过程的特性, 直线的斜率越大, 表 明阻抗的虚部增大, 反映了该电化学体系的电容行 为更加突出. 从图中可见, RCAs 的 Nyquist 曲线(b) 在低频区域直线的斜率大于 CAs 的 Nyquist 曲线(a) 中直线的斜率, 表明经过结构重整后, 电解液更容 易在电极材料中扩散, 从而改善了它的电容性能. 在高频区, Warburg 阻抗变得不重要, 电解质溶液的 欧姆电阻和电荷传递电阻变成电化学体系中的重 要组成, 在 Nyquist 曲线中, 表示为曲线的圆弧部分. 圆弧右端的延长弧线与阻抗实部坐标轴相交处的 数值, 表示该电化学体系阻抗的实部. 从图中可见, 由 RCAs 作电极材料组成的电化学体系的阻抗, 明 显小于由 CAs 作为电极材料组成的电化学体系, 说

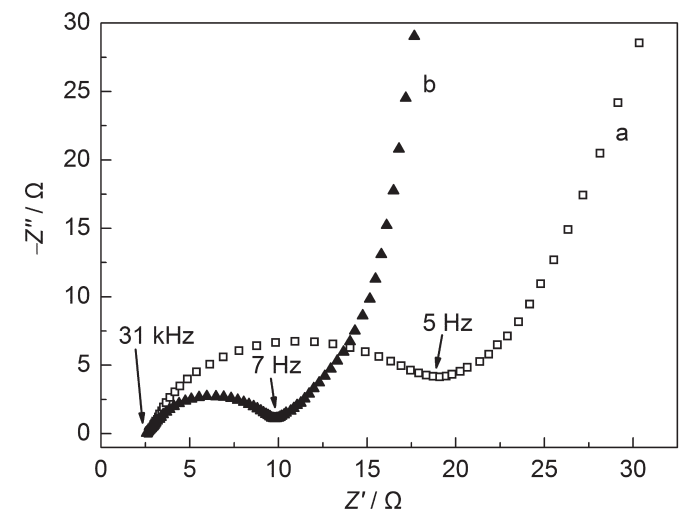

图 6 CAs (a) 和 RCAs (b) 的交流阻抗谱

Fig.6 AC impedance spectroscopies of CAs (a) and RCAs (b) 


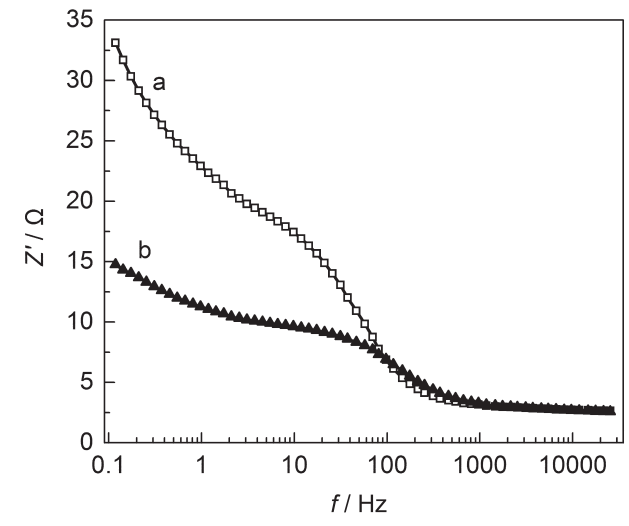

图 7 CAs (a) 和 RCAs (b) 的电极阻抗与频率变化关系曲线 Fig.7 Dependence of impedance of CAs (a) and RCAs (b) electrodes on frequency

明电荷在 RCAs 骨架上的传递更加容易. 这是由于 CAs 经过结构重整以后, 其中的无定形碳纳米颗粒 发生定向重排, 形成层状碳的结构, 使其导电能力 有所提高, 同时结构重整引起材料孔结构和孔径分 布的改变, 有利于电解质溶液在电极材料中的扩 散. 继续增大交流频率, 电极/溶液界面对高频没有 响应, 此时电化学体系的阻抗只来自电解质溶液的 欧姆电阻, 因此, 图中两条曲线中圆弧的左端部分 相交, 此时的交流频率约为 $31 \mathrm{kHz}$.

\subsubsection{CAs 和 RCAs 的 Bode 曲线}

以电化学阻抗谱测试过程中使用的频率信号 为 $X$ 轴, 对应的阻抗为 $Y$ 轴作图可以得到 Bode 曲 线, 从 Bode 曲线可以直接得到电极/溶液电化学体 系的频率响应特性. CAs 和 RCAs 电极/溶液的 Bode 曲线如图 7 所示. 由图中可见, 将 RCAs 与 CAs 作为 电极材料的电化学体系比较, 前者在中、低频段, 显 现出较低的阻抗和较高的截止频率(达到平台时的 频率), 表明 RCAs 作为电极材料在较宽的交流频率 变化范围内, 电容器的性能可保持稳定, 有益于扩 展电容器的应用范围.

\section{4 结 论}

CAs 在金属钠熔体中发生结构重整, 所得 RCAs 样品在 2 和 $4 \mathrm{~nm}$ 的孔径处分别有较为集中的 孔径分布, 而且孔径小于 $10 \mathrm{~nm}$ 以下的孔体积占其 总孔容的比例为 $30 \%$, 比表面积增加约 48\%; 由 RCAs 为电极材料组成的电化学体系的阻抗约为由 CAs 为电极材料组成的电化学体系阻抗时的 $45 \%$, 电容性能比较稳定; RCAs 的网络骨架有利于电解 质在其中的扩散与渗透, 使得 RCAs 比 CAs 更容易 保持电容特性.
致谢：本文实验研究过程中的部分分析测试工作得到同济 大学化学系实验中心的支持.

\section{References}

(1) Hou, C. H.; Liang, C. D.; Yiacoumi, S.; Dai, S.; Tsouris, C. J. Colloid Interface Sci. 2006, 302, 54.

(2) Li, J.; Wang, X. Y.; Huang, Q. H.; Dai, C. L. The Chinese Journal of Nonferrous Materials 2006, 16, 1468. [李 俊, 王先友, 黄庆华, 戴春玲. 中国有色金属学报, 2006, 16, 1468.]

(3) Frackowiaka, E.; Beguin, F. Carbon 2001, 39, 937.

(4) Bordjiba, T.; Mohamedi, M.; Dao, L. J. Power Sources 2007, 172, 991 .

(5) Amini, N.; Aguey-Zinsou, K. F.; Guo, Z. X. Carbon 2011, 49, 3857.

(6) Huang, C. H.; Doong, R. A.; Gu, D.; Zhao, D. Y. Carbon 2011, 49, 3055 .

(7) Zhou, J. H.; He, J. P.; Ji, Y. J.; Zhao, G. W.; Zhang, C. X.; Chen, X.; Wang, T. Acta Physico-Chimica Sinica 2008, 24, 839. [周 建华, 何建平, 计亚军, 赵桂网, 张传香, 陈 秀, 王 涛. 物理 化学学报, 2008, 24, 839.]

(8) Ji, Y. J.; He, J. P.; Zhou, J. H.; Dang, W. J.; Liu, X. L.; Zhang, C. X.; Zhao, G. W. J. Mater. Sci. Eng. 2007, 4, 501. [计亚军, 何建平, 周建华, 党王娟, 刘晓否, 张传香, 赵桂网. 材料科学 与工程学报, 2007, 4, 501.]

(9) Francisco, J. M. H.; Maldonado, H.; Carlos, M. C.; Francisco, C. M.; Agustín F. J. Hazard. Mater. 2007, 148, 548.

(10) Feng, Y.; Miao, L.; Tanemura, S.; Tanemura, M.; Suzuki, K. Rare Metals 2006, 25, 284.

(11) Pekala, R. W.; Alviso, C. T.; Lu, X.; Grob, J.; Fricke, J. J. NonCryst. Solids 1995, 188, 34.

(12) Wu, D. C.; Fu, R. W.; Dresselhaus, M. S.; Dresselhaus, G. Carbon 2006, 44, 675.

(13) Zhai, D. Y.; Du, H. D.; Li, B. H.; Zhu, Y.; Kang, F. Carbon 2011, 49, 718.

(14) Wang, Z. L.; Zhang, X. B.; Liu, X. J.; Lv, M. F.; Yang, K. Y.; Meng, J. Carbon 2011, 49, 161.

(15) Srinivasa, G.; Lovellb, A.; Howarda, C. A.; Skippera, N. T.; Ellerbya, M.; Bennington, S. M. Synthetic Metals 2010, 160, 1631.

(16) Claire, H.; Albert, H.; Philippe, L. Solid State Sciences 2004, 6, 125.

(17) Pelleg, J.; Ashkenazi, D.; Ganor, M. Mater. Sci. Eng. A 2000, 281,239

(18) Xu, Z. J.; Ji, T.; Wang, W. Y.; Xia, B. Z.; Ma, C.; Gan, L. H. Acta Physico-Chimica Sinica 2011, 27, 262. [徐子颉, 吉 涛, 王玮衍, 夏炳忠, 马 超, 甘礼华. 物理化学学报, 2011, 27, 262.]

(19) Tuinstra, F.; Koenig, J. L. J. Chem. Phys. 1970, 53, 1126.

(20) Nakamizo, M.; Kammereck, R.; Walker, P. L. Carbon 1974, 12, 259.

(21) Nikiel, L. Carbon 1993, 31, 1313 